\title{
MEMS Variable Capacitor Using Cross Membranes for RF Band
}

\author{
Takeaki Shimanouchi Non-member (Fujitsu Ltd., shimanouchi@jp.fujitsu.com) \\ Masahiko Imai Non-member (Fujitsu Ltd., imai.masahiko@jp.fujitsu.com) \\ Tadashi NakataniＮon-member（Fujitsu Ltd., tnakatani@jp.fujitsu.com) \\ Anh Tuan Nguyen Non-member (Fujitsu Laboratories Ltd., ngtuananh@jp.fujitsu.com) \\ Satoshi Ueda Non-member (Fujitsu Ltd., ueda_satoshi@jp.fujitsu.com) \\ Ippei Sawaki Member (Fujitsu Laboratories Ltd., sawaki@jp.fujitsu.com) \\ Yoshio Satoh Member (Fujitsu Ltd., satoh.yoshio@jp.fujitsu.com)
}

Keywords : variable capacitor, quality factor, RF

Variable capacitors are necessary as tuning elements in the RF front-ends of mobile communication systems. Tuning element applications include phase shifters, tunable filters, impedance matching circuits, voltage controlled oscillators, etc.

Currently, solid-state variable capacitors have disadvantages such as low quality factor and low electrical resonant fequency. Therefore, MEMS variable capacitors are highly expected because of their high quality factor and high electrical resonant fequency.

Generally reported MEMS variable capacitor is gap tuning type that is achieved by tuning the gap between two parallel plate electrodes. One electrode is fixed on a substrate and the other one is a movable electrode. The conventional capacitor using electrostatic force is very simple structure. However, with this type of capacitor, pull-down phenomenon happens when the gap between two parallel plates become less than $2 / 3$ of the initial gap. At this position, the electrostatic force becomes higher than the mechanical restoring force and the movable electrode will be suddenly pulled down to the fixed electrode. The gap during this pull-down movement cannot be controlled. Therefore the minimum gap between two electrodes is $2 / 3$ of the initial gap. In other word, the maximum tuning range of the capacitor is only $150 \%$.

In this paper, we describe a novel design of MEMS variable

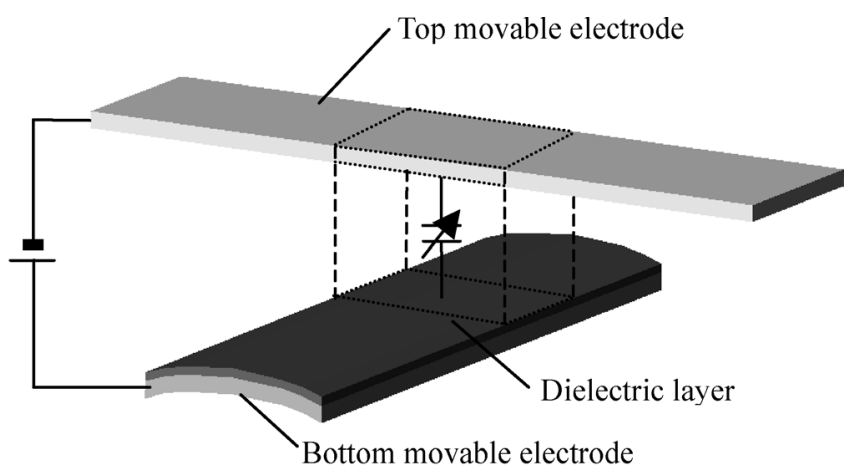

Fig. 1. Schematic diagram of the proposed capacitor capacitor with high quality factor and wide tuning range that based on the gap tuning capacitor.

Figure 1 shows a schematic diagram of the proposed MEMS variable capacitor. The top and bottom movable electrodes cross each other, and the bottom electrode has a dielectric layer on the side of the top electrode. The bottom electrode deformes convexly towards the top electrode. When bias voltage is applied to two electrodes, electrostatic force pulls them inwards, causes change in the gap and the capacitance.

A photograph of the variable capacitor is shown in Figure 2. The chip size is $1.5 \times 1.8 \mathrm{~mm}^{2}$. In this variable capacitor, the top electrode is ground like a varactor diode. $50 \Omega \mathrm{CPW}$ is composed of the signal line and the ground.

The variable capacitor has tuning range of $330 \%$ at $5 \mathrm{~V}$. High quality factor of 52 at $2 \mathrm{pF}$ and $2.4 \mathrm{GHz}$ was also achieved. The electrostactic working principle and low operating voltage enables nearly zero power consumption. The fabrication process using only basic surface processing is expected to increase the productivity and reduce the device costs.

This research was supported by the National Insitute of Information and Communications Technology (NICT) of Japan.

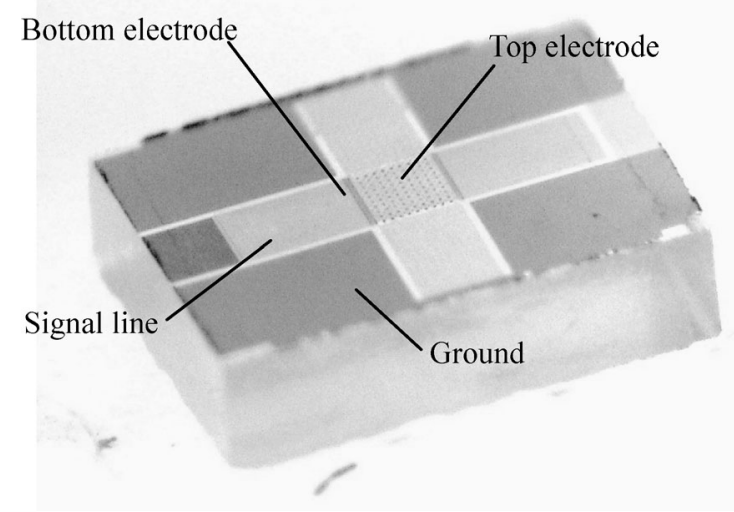

Fig. 2. Photograph of proposed variable capacitor 


\section{MEMS Variable Capacitor Using Cross Membranes for RF Band}

$\begin{array}{ll}\text { Takeaki Shimanouchi* } & \text { Non-member } \\ \text { Masahiko Imai* }^{*} & \text { Non-member } \\ \text { Tadashi Nakatani* } & \text { Non-member } \\ \text { Anh Tuan Nguyen** } & \text { Non-member } \\ \text { Satoshi Ueda* } & \text { Non-member } \\ \text { Ippei Sawaki** } & \text { Member } \\ \text { Yoshio Satoh* } & \text { Member }\end{array}$

This paper describes a novel design of MEMS variable capacitor with high quality factor and wide tuning range. The proposed MEMS variable capacitor is composed of two cross movable electrodes and one dielectric layer between them. The fabrication process does not require any complex processes, such as wafer transfer, wafer backside etching, special planarization or supercritical point drying. The capacitor has tuning range of $330 \%$ at $5 \mathrm{~V}$ bias voltage, and an overall quality factor of 52 at $2 \mathrm{pF}$ and $2.4 \mathrm{GHz}$.

Keywords : variable capacitor, quality factor, RF

\section{Introduction}

Variable capacitors are necessary as tuning elements in the RF front-ends of mobile communication systems. Tuning element applications include phase shifters, tunable filters, impedance matching circuits, voltage controlled oscillators, etc.

Currently, solid-state variable capacitors have disadvantages such as low quality factor and low electrical resonant fequency. Therefore, MEMS variable capacitors are highly expected because of their high quality factor and high electrical resonant fequency. There are three most commone types of MEMS variable capacitor. The first type is gap tuning capacitor that is achived by tuning the gap between two parallel plate electrodes ${ }^{(1)(2)}$. One electrode is fixed on a substrate and the other one is a movable electrode. The conventional capacitor using electrostatic force is very simple structure. However, with this type of capacitor, pull-down phenomenon happens when the gap between two parallel plates become less than $2 / 3$ of the initial gap. At this position, the electrostatic force becomes higher than the mechanical restoring force and the movable electrode will be suddenly pulled down to the fixed electrode. The gap during this pull-down movement cannot be controlled. Therefore the minimum gap between two electrodes is $2 / 3$ of the initial gap. In other word, the maximum tuning range of the capacitor is only $150 \%$.

Recently, some capacitors with wide tuning range were reported $^{(3)(4)}$. Unfortunately, these capacitors have low quality factor at RF band. The second type is area tuning capacitor that is achived by tuning overlapping area using comb drive actuator ${ }^{(5)}$. This type of capacitor has wide tuning range, but has large size. On the other hand, in order to archiving low driving voltage, this variable capacitor must employ small beams. This leads to large equivalent series resistance (ESR) of the beams and causes low

* Fujitsu Ltd.

** Fujitsu Laboratories Ltd.

64, Nishiwaki, Ohkubo-cho, Akashi 674-8555 quality factor. The third type is switched capacitor that obtaines the variable capacitance by selecting fixed capacitance using switches ${ }^{(6)}$. This type has very flexible tuning range but again has larger size and needs complex control circuits.

Consumer-requested features for tuning applications include high quality factor, wide tuning range, low power consumption, and low driving voltage. There are no MEMS variable capacitors known to the authors have obtained all of these features. It is especially difficult to achieve a high quality factor in the RF range because the quality factor is inverse-proportional to the frequency.

In this paper, the design and fabrication of an electrostatic type MEMS variable capacitor based on the gap tuning capacitor will be presented. In order to achieve high quality factor, the capacitor is fabricated on glass substrate and both electrodes are movable membranes. The capacitor has wide electrodes, which help to minimize the ESR, and contribute to high quality factor. A thin dielectric layer on top of bottom electrode allows very close distance between two electrodes that strategically increases the tuning range of the capacitor and decreases the driving voltage.

The tuning range of $330 \%$ and quality factor of 52 at $2 \mathrm{pF}$ and $2.4 \mathrm{GHz}$ was achieved by this structure. The driving voltage is only $5 \mathrm{~V}$.

\section{Working Principle of Variable Capacitor}

Figure 1 shows a schematic diagram of the proposed MEMS variable capacitor. The top and bottom movable electrodes cross each other, and the bottom electrode has a dielectric layer on the side of the top electrode. The bottom electrode deformes convexly towards the top electrode. When bias voltage is applied to two electrodes, electrostatic force pulls them inwards, causes change in the gap and the capacitance.

The relative positions of two electrodes are shown in Figure 2. When the applied bias voltage between the top and bottom electrodes increases, at first the electrodes come closer under electrostatic force, next they contact at the dielectric layer, and 
finally they stick together.

The capacitor has two stages of non-contact and contact. Mechanically, this structure also has two types of springs. One is the electrode membrane and another one is the deformation caused by curvature difference between the top electrode and the bottom electrode. Before the top and bottom electrodes contact, the electrostatic force balances with the membrane springs of top and bottom electrodes. After the top and bottom electrodes contact, the electrostatic force balances with both membrane and deformation spring.

The capacitance $\mathrm{C}$ between the two electrodes is gived by:

$$
C=\frac{\varepsilon_{0} \varepsilon_{r} S}{d}
$$

where $\varepsilon_{0}$ is the dielectric constant of a vaccum, $\varepsilon_{r}$ is relative dielectric constant, $S$ is the area of capacitor electrode, $d$ is the gap between the two electrodes. When there are a dielectric layer and an air layer between the electrodes, we have following relationship:

$$
\frac{d}{\varepsilon_{r}}=\frac{d_{\text {air }}}{\varepsilon_{\text {air }}}+\frac{d_{\text {dielectric }}}{\varepsilon_{\text {dielectric }}}
$$

We can see that the larger the change of the gap between electrodes, the higher the capacitance change or wider tuning range. However to avoid pull-down, a gap equals to $2 / 3$ of the initial gap is the lower limit for conventional type capacitor. The current capacitor with the dielectric layer can operate even when two electrodes contact. When two electrodes are in contact, the convex electrode prevents pull-down phenomenon to occur. Consequently, the gap of the capacitor can be precisely controlled from initial value down to zero, producing a very wide tuning range.

The electrostatic force $F$ between the two electrodes is gived

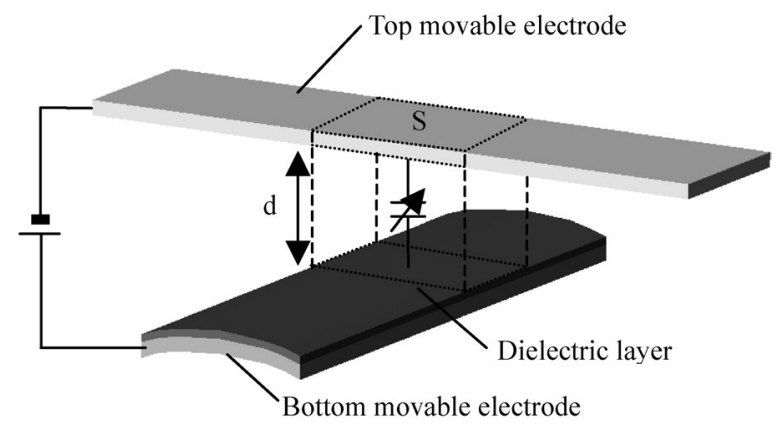

Fig. 1. Schematic diagram of the proposed capacitor

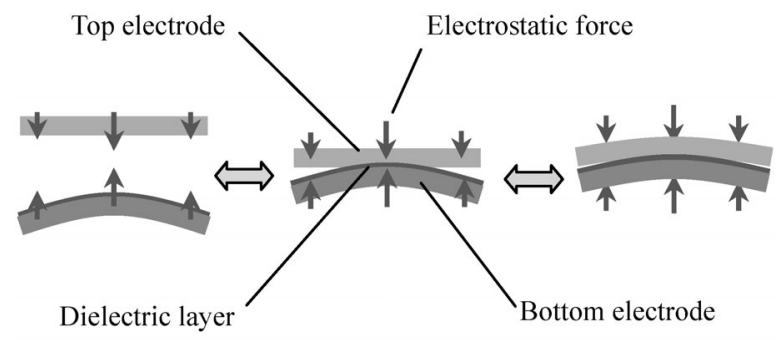

Small $\longrightarrow$ Bias voltage

Fig. 2. Relative position between two electrodes when bias voltage changes by:

$$
F=\frac{S}{2 d^{2}} \varepsilon_{0} \varepsilon_{r} V^{2}
$$

where $V$ is the bias voltage between two electrodes. From this equation, we also see that narrow gap enables large electrostatic force at low driving voltage and small electrode area.

\section{Design and Fabrication Process}

Figures 3 shows an exploded diagram of the proposed variable capacitor. The structure has 5 layer groups: glass substrate, $\mathrm{SiO}_{2}$ insulation layer, bottom movable electrode with dielectric layer, top movable electrode layer and bonding pad layer. The top and bottom electrodes stand on substrate themselves. The signal transmission lines (the bottom electrodes without an overlapping area) is an air coplanar-waveguide (CPW) structure because they have ground electrodes on both sides. Therefore the dielectric loss due to the substrate and the transmission loss can be efficiently reduced.

We carried out the electrical and mechanical simulaton of the proposed variable capacitor by CoventorWare. The capacitor is simply modeled by two electrodes without etching holes, and a dielectric layer. Figure 4 shows the deformation of the top and bottom movable electrodes, the bottom electrode deformes covexly towards the top electrode because of the difference of internal stresses between the dielectric layer and the bottom electrode.

Figure 5 shows the relationship between capacitance and applied voltage. When the bias voltage changes from $0 \mathrm{~V}$ to $5 \mathrm{~V}$, the capacitance changes from $1.5 \mathrm{pF}$ to $4.7 \mathrm{pF}$. The corresponding tuning range is $310 \%$.

Fig. 6 shows the contact area and contact force between two electrods as function of applied voltage. It is obvious that the contact area becomes non-zero, also means electrodes go in

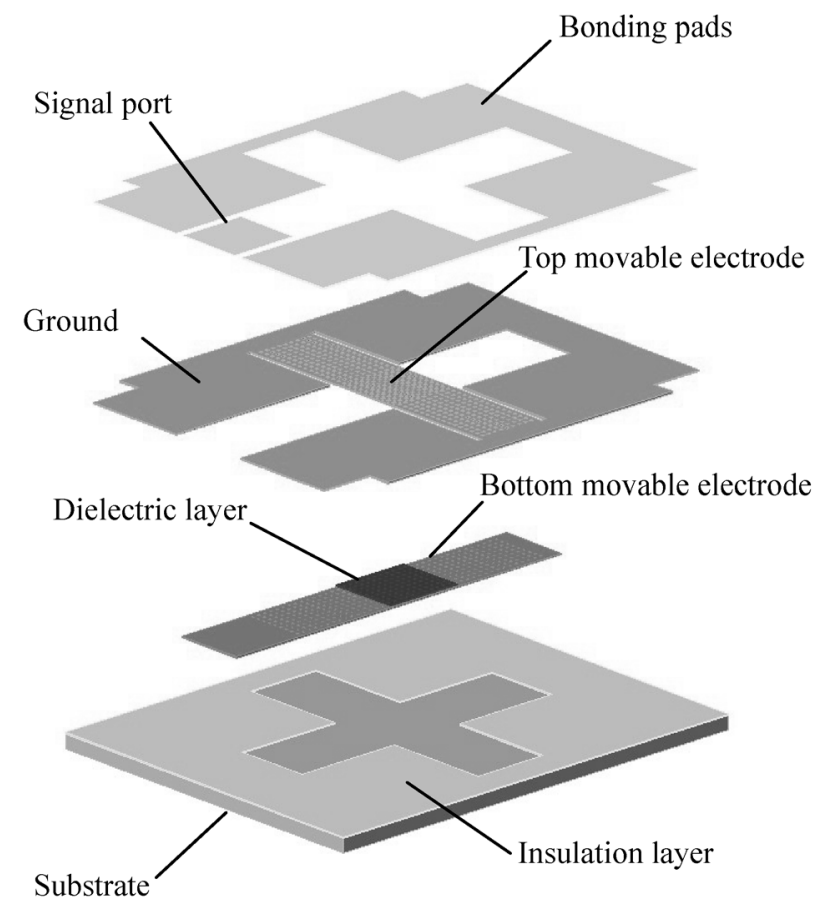

Fig. 3. Exploded diagram of the proposed variable capacitor 


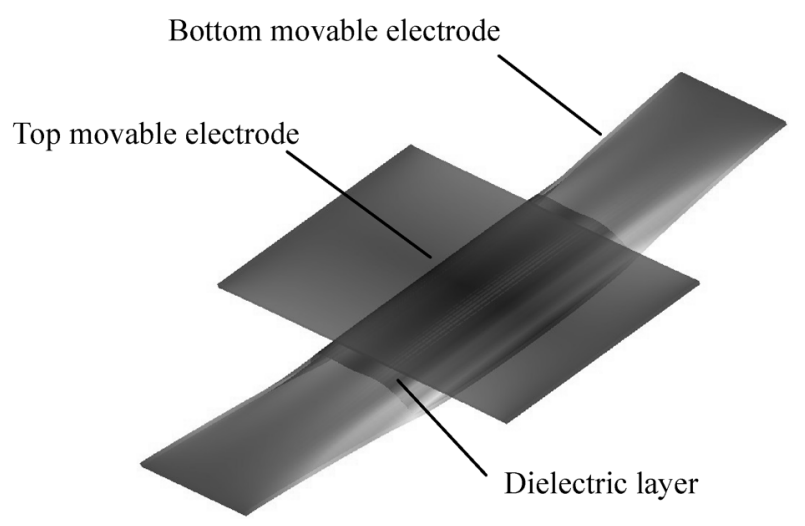

Fig. 4. Deformation of top and bottom electrodes

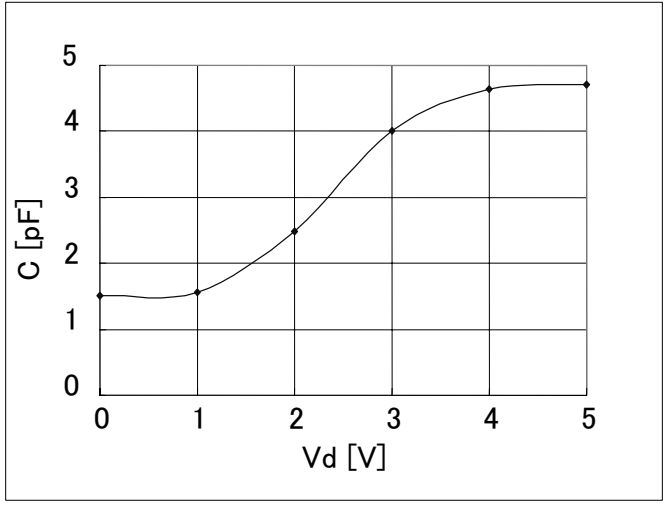

Fig. 5. The simulated tuning range

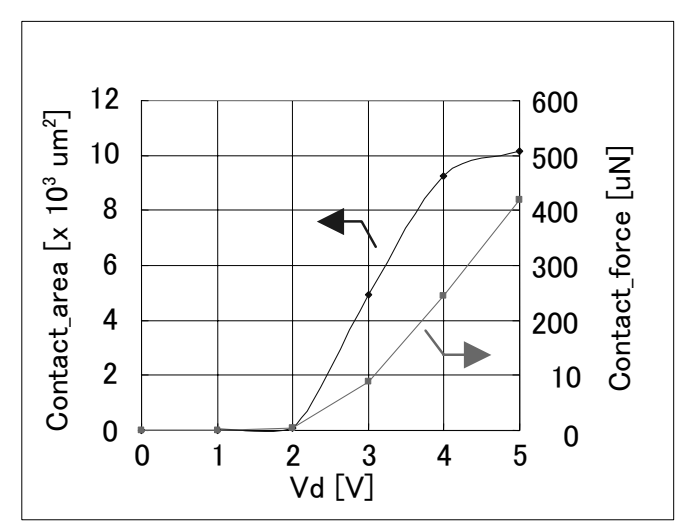

Fig. 6. The simulated contact area and contact force

contact at about $2 \mathrm{~V}$ bias volatge. The capacitor has separate stage from $0 \mathrm{~V}$ to about $2 \mathrm{~V}$ and contact stage from about $2 \mathrm{~V}$ to $5 \mathrm{~V}$. The simulated mechanical resonant frequency is over $270 \mathrm{kHz}$, which is very suitable for mobile applications.

The fabrication process of the variable capacitor is shown in Figure 7. All the processes are pure surface processing.

First, insulation layer of PECVD silicon oxide is deposited onto glass wafer. Cavity for separating the bottom electrode and the substrate is formed by dry etching process, and silicon sacrificial layer 1 is sputtered into the cavity. (Figure 7a) .

Next, aluminum bottom electrode and thin aluminum oxide dielectric layer are formed by sputtering method. (Figure 7b)

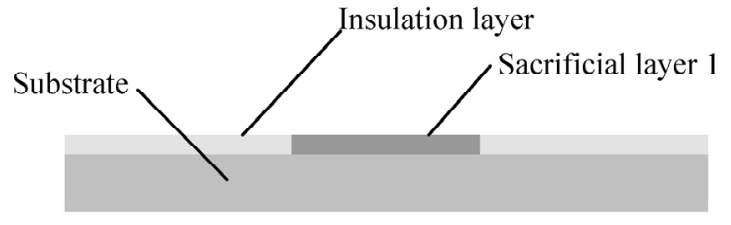

(a) Insulation layer and sacrificial layer formation

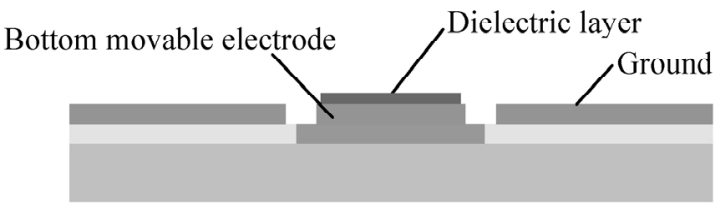

(b) Bottom electrode and dielectric layer formation

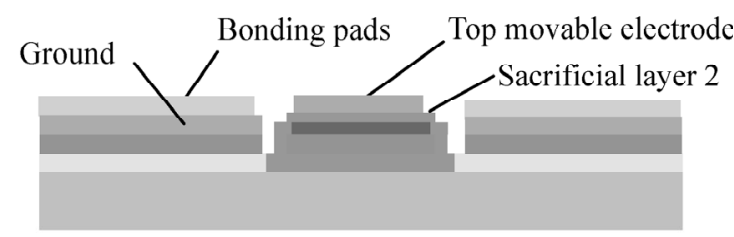

(c) Sacrificial layer and top electrode formation

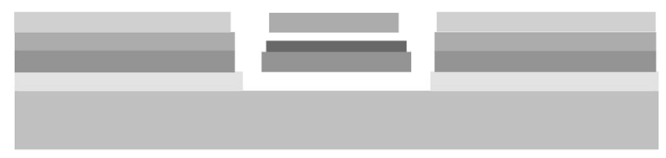

(d) Bonding pad formation and sacrificial layer etching

Fig. 7. Fabrication process of proposed variable capacitor

The second silicon sacrificial layer 2, aluminum top movable electrode, and gold contact pads are desposited and patterned in order. (Figure 7c)

Finally, the sacrificial layers 1 and 2 are released by dry-etching using $\mathrm{SF}_{6}$ gas. The special process such as supercritical point drying is not necessary. (Figure 7d).

\section{Experimental Results}

A photograph of the variable capacitor is shown in Figure 8. The chip size is $1.5 \times 1.8 \mathrm{~mm}^{2}$. In this variable capacitor, the top electrode is ground like a varactor diode. $50 \Omega \mathrm{CPW}$ is composed of the signal line and the ground.

A SEM photo of the cross membranes area is shown in Figure 9. The two electrodes have holes for accelerating the release of the capacitor.

The RF characteristics of the capacitor was measured by using vector network analyzer and coplaner probe.

Figure 10 shows the relationship of the bias voltage and the capacitance at $2.4 \mathrm{GHz}$. The capacitance is tuned from 1.80 to $6.02 \mathrm{pF}$ with a $5 \mathrm{~V}$ bias voltage. The tuning rage is about $330 \%$, and this transition does not exhibit any hysteresis.

Figure 11 shows the return loss $\left(S_{I I}\right)$ on the capacitor Smith chart from $300 \mathrm{MHz}$ to $8 \mathrm{GHz}$. The overall quality factor calculated from this measured data is 52 at $2 \mathrm{pF}$ and $2.4 \mathrm{GHz}$. The resonant frequency is about $8 \mathrm{GHz}$.

We think that the differences between experimental results and simulation results are caused by the influence of the parasitic capacitance and the internal stress of each layers such as two movable electrodes, dielectric layer and insulation layer. 


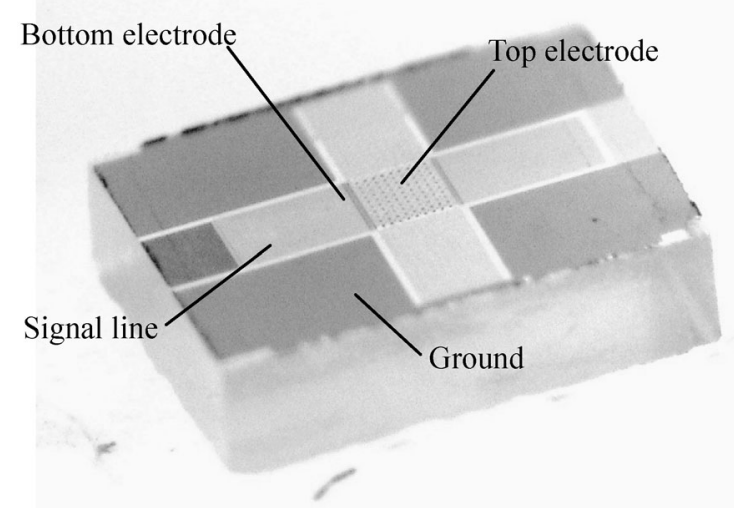

Fig. 8. Photograph of proposed variable capacitor

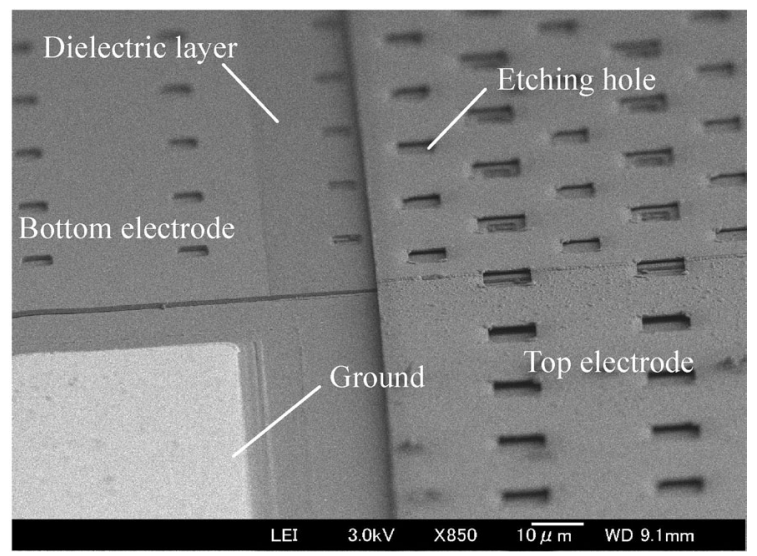

Fig. 9. SEM of the cross membrane area

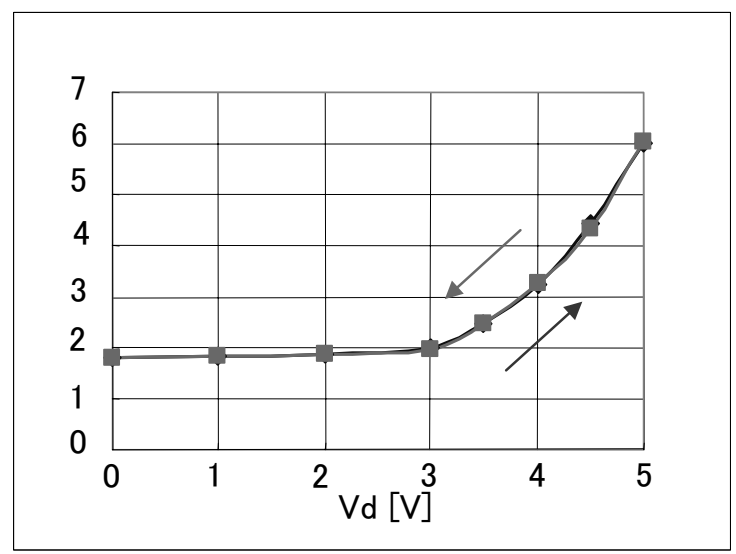

Fig. 10. Relationship of bias voltage and capacitance

\section{Conclusions}

Variable capacitor with high quality factor and wide tuning range was designed and fabricated. The fabricated variable capacitor has tuning range of $330 \%$ at $5 \mathrm{~V}$. High quality factor of 52 at $2 \mathrm{pF}$ and $2.4 \mathrm{GHz}$ was also achieved. The electrostactic working principle and low operating voltage enables nearly zero power consumption. The fabrication process using only basic surface processing is expected to increase the productivity and reduce the device costs.

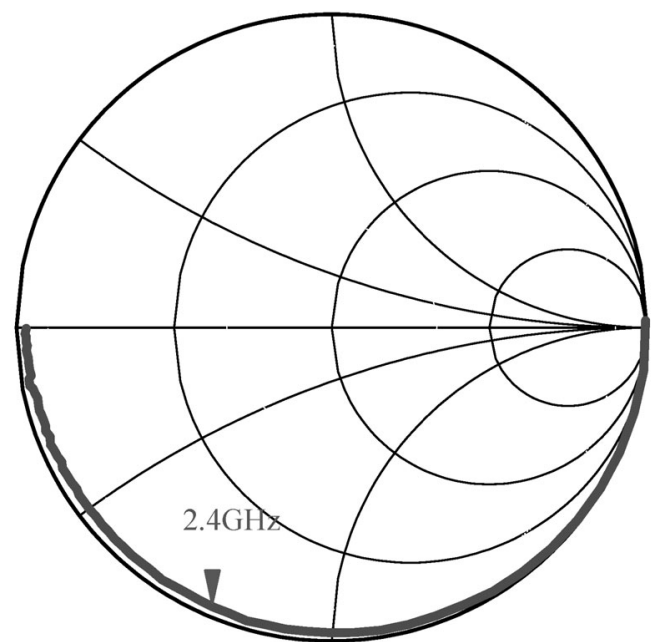

Fig. 11. Return loss at $2 \mathrm{pF}$

\section{Acknowledgement}

This research was supported by the National Institute of Information and Communications Technology (NICT) of Japan.

(Manuscript received Aug.26, 2005, revised Nov. 16, 2005)

\section{References}

(1) D. J. Young and B. E. Boser : "A micromachined variable capacitor for monolithic low-noize VCOs,", Tech. Digest. Solid State Sensor and Actuator Workshop, pp.86-89 (1996)

(2) A. Dec and K. Suyama : "Micromachined electro-mechanically tunable capacitors and their applications to TF IC's", IEEE Trans. Microwave Theory Tech., Vol.46, No.12, pp.2587-2595 (1998-12)

(3) M. Bakri-Kassem and Raafat R. Mansour : "An Improved Design for Parallel Plate MEMS Varible Capacitors", Microwave Symposium Digest, 2004 IEEE MTT-S, pp.865-868 (2004)

(4) D. Peroulis, Y. Lu, and L. P. B. Katehi : "Highly Reliable Analog MEMS Varavtors", Microwave Symposium Digest, IEEE MTT-S, pp.869-872 (2004)

(5) C. Tsai, et al. : "An Isolated Tunable Capacitor with a Linear Capacitance-Voltage Behavior", Transducers, pp.833-836 (2003)

(6) J. Brank, J. Yao, M. Eberly, A. Malczewski, K. Varian, and C. L. Goldsmith, : "RF MEMS-based tunable filters", Int. J. RF Microwave CAE, Vol.11, pp.285-300 (2001)

Takeaki Shimanouchi (Non-member) was born in Kochi Prefecture,

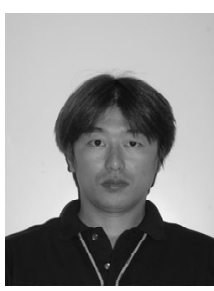
Japan, in 1965. He received the B.E. and M.E. degrees, in department of materials science from Toyohashi University of Technology in 1988 and 1990. In 2000 he joined Fujitsu Laboratories LTD., where he was engaged in research and development of micro actuators. Currently, he is researching RF-MEMS devices. Besides working for Fujitsu Laboratories LTD., he is also working for Fujitsu

LTD. in the same field.

Masahiko Imai

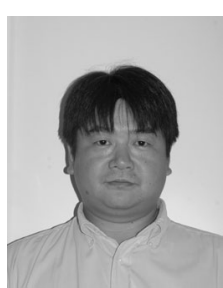

(Non-member) was born in Hyogo Prefecture, Japan in 1967. He graduates from Himeji technical high school in 1986. In 1986 he joined Fujitsu Laboratories LTD., where he was engaged in research and development of semiconductor devices and SAW devices. Currently, he is researching RF-MEMS devices. Besides working for Fujitsu Laboratories LTD., he is also working for Fujitsu LTD. in the same field. 
Tadashi Nakatani (Non-member) was born in Hyogo Prefecture, Japan on February 6, 1968. He received the B.E. and M.E. degrees, in the field of electrical engineering from Kobe University in 1990 and 1992. In 1992 he joined Fujitsu Laboratories LTD., where he was engaged in research and development of field emission display and SAW devices. Currently, he is researching MEMS devices. Besides working for Fujitsu Laboratories LTD., he is also working for Fujitsu LTD. in the same field.

\section{Anh Tuan Nguyen}

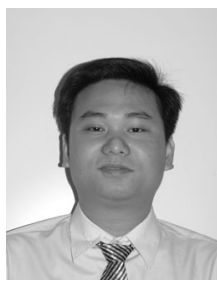

(Non-member) was born in Hung Yen, Viet Nam on May 24, 1978. He received the B.E. degree in aeronautical engineering from Ho Chi Minh University of Technology, Vietnam in 2001 and M.E. degree in information science and systems engineering from Ritsumeikan University, Japan in 2003. He joined Fujitsu Laboratories in 2004 and currently works on RF-MEMS devices.

\section{Satoshi Ueda}

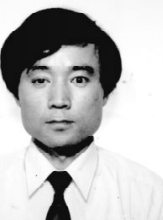

(Non-member) was born in 1954. He received the M.E. degrees, in the field of electrical engineering from Kobe University in 1979. In 1979 he joined Fujitsu Laboratories LTD., where he was engaged in research and development of IR sensor. Currently, he is researching MEMS devices. Besides working for Fujitsu Laboratories LTD., he is also working for Fujitsu LTD. in the same field.

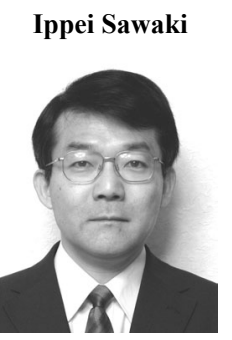

(Member) was born in 1956. He received the M.E. degrees from Tokyo University in 1980. In 1980 he joined Fujitsu Laboratories LTD., where he was engaged in research and development of optical waveguide devices, optical waveguided SHG and head of optical magnetic disk drives. Currently, he is researching MEMS devices. He is a member of The Japan Society of Applied Physics.
Yoshio Satoh

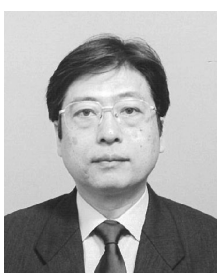

(Member) was born in Niigata Prefecture, Japan, in 1949. He received the B.S. and M.S. degree in electronics engineering from Tohoku University, Sendai, Japan, in 1972 and 1974, respectively. He received the Ph.D. degree in communication engineering from Tohoku University in 1996. In 1974 he joined Fujitsu Laboratories LTD., where he was engaged in research and development of the bubble memory devices. Since 1989, he was engaged in research and development of RF micro devices such as SAW filters, FBAR filters and RF-MEMS devices. Besides working for Fujitsu Laboratories LTD., he is also working for Fujitsu LTD. in the same field. He received the medal with purple ribbon from the Japanese Government for developments of SAW filters in 2003. 